\title{
Could Computer Game Players React as Quick as Table Tennis Athletes and Perform the Right Action?
}

\author{
Senay Koparan Sahin ${ }^{1}$, Engin Sagdilek ${ }^{2 *}$
}

\begin{abstract}
Objective: Table tennis athletes have short reaction times and specialized skills. Computer games may also decrease reaction times. However the actions of the computer game players are limited to mouse/keyboard/joystick use. The objective of the study is to compare the reaction times and the achievement levels on selective action array of athletes and players.
\end{abstract}

Material and Methods: 10 medical school students that played computer games 10 hours a week for at least 5 years, and 9 table tennis athletes that were trained 10 hours a week for at least 5 years were included in the study. Right/left-hand/foot reaction times against auditory and visual stimuli were recorded for each subject. Selective action array was implemented utilizing a table tennis robot. The robot was set up to send 120 balls in different colors, to different points on the table, in random colors and 90 balls per minute at the same speed. The subjects were asked to ignore the white balls, to touch the yellow balls and to hold the pink balls. The actions that the subjects took, or did not take against each ball were transformed into points.

Results: It was found that the mean reaction time for athletes was $196.8 \mathrm{~ms}$ and $196.0 \mathrm{~ms}$ for the players. The selective action array total points were similar in athletes and players. While the athletes scored better with yellow and pink balls, players scored better with the white balls.

Conclusion: The similar scores and reaction times by players and athletes could be interpreted that it could be beneficial to prioritize visual perception, attention and focusing in selecting athletes and in designing training programs.

Key words: Computer games, table tennis, reaction time, selective action array

\section{Introduction}

Table tennis is one of the sports that are technically difficult to learn and play. The athlete has to perceive the ball within a small space, in various rotations, aiming at different points on the table in various speeds, and perform the correct action to send the ball to the opponent's side to force him to fail. This sport where the ball could reach high speeds within a range of 3-5 $\mathrm{m}$ is considered among reaction sports (1).

Table tennis table is 152.5 by $274 \mathrm{~cm}$ and the diameter of the table tennis ball is $40 \mathrm{~mm}$ and it weighs 2.7 grams. Measurements taken with professional athletes demonstrated that the maximum speed of the ball could reach $31 \mathrm{~m} / \mathrm{s}$ (2), and with that speed it could traverse the table within approximately $90 \mathrm{~ms}$. With an average speed of $10 \mathrm{~m} / \mathrm{s}$, the time that the ball covers the length and back between two athletes that stand $30 \mathrm{~cm}$ from the table is approximately $300 \mathrm{~ms}$.
Although the maximum speed that the ball could reach in table tennis is almost half the speed it could reach in other sports played with racket such as tennis, squash and badminton, the relatively shorter space between the two athletes in table tennis requires a relatively shorter time of reaction by table tennis athletes when compared to other racket sports athletes and many other sports athletes and to individuals that do not participate in sports (1,3-6).

Computer games play an increasingly important part in our lives and became one of the most prevalent entertainment tools of the younger generation. During recent years, international tournaments for computer games are organized and even the term electronic athlete / e-athlete is being used in the literature (7-11).

The intrinsic concept of movement in sports does not completely exist in electronic sports, which became the main topic of the argument on whether these games should be considered as sports at all.

Received 13-11-2015, Accepted 22-12-2015, Available Online 15-02-2016

1 Uludag University, Sport Science Faculty, Dept. of Physical Education and Sport Teaching Bursa-Turkey

2 Uludag University, Faculty of Medicine, Dept. of Biophysics Bursa-Turkey

*Corresponding Author: Engin Sagdilek E-mail: esagdilek@hotmail.com 
Success in computer games is related to attention and concentration. Especially in action games, it is an expected fact that the visual perception of the players should be advanced and their reaction times should be short. However, the actions of these players could not reach beyond the use of mouse, keyboard and joystick. The objective of the study is to compare the reaction times for advanced level table tennis athletes and computer game players and the accuracy rates of the required actions utilizing a new test to determine selective reactions of table tennis athletes and computer game players

\section{Material and Methods}

The study was conducted in Uludağ University, Faculty of Sport Sciences, during the months of March and April 2013. An approval was obtained from the "Uludağ University, Faculty of Medicine, Clinical Research Ethics Board". The subjects were included in the study after obtaining their consent.

\section{Study Groups}

Professional or amateur table tennis athletes, who were licensed in various table tennis clubs in Bursa, Turkey, were provided information on the study and participated in the study on a voluntary basis. The criterion for acceptance in the study was at least 10 hours training per week for at least 5 years. 9 table tennis players with a mean age of 20.1 years that met the criterion participated in the study [Table Tennis Athletes Group (TT)].

Computer game players that met similar conditions with the table tennis athletes were scanned among university students and it was found that mostly medical students met the criterion. Instead of forming a mixed group from different departments, a homogenous group was formed including only Faculty of Medicine students. Since the objective of the study was to compare advanced table tennis athletes and computer game players, educational status of table tennis athletes and computer game players was not considered as a significant issue for the assessed parameters and assessment methods. An announcement was made for freshmen, sophomore and junior Faculty of Medicine students and volunteers that met the study criteria were included in the study. 10 computer game players with a mean age of 19.6 years, who were playing computer games for at least 10 hours per week for at least 5 years participated in the study [Computer Game Players Group (CG)].

It was made sure that the subjects did not suffer from an active disease and the tests were implemented at the same hour of the day. Subjects with acute infections or had taken alcohol within one day of the tests were excluded from the study.

Medical Science and Discovery 2016; 3(2): 91-7

\section{Reaction Time Measurements}

Reaction time was measured using specialized equipment produced for these tests. This equipment consisted of a button to enter the stimulus, a second button to determine the type of the stimulus (sound or light) and a digital screen where the reaction time could be read. Sound stimulus was given using a loudspeaker mounded on the equipment; light stimulant was given by a LED lamp, connected to the equipment with cables and mounted on a support that sits exactly at the opposite of the subject. At one end of the apparatus shaped like a "U" sits the light source and at the other end, there is an optic sensor. Placing the hand or foot of the subject in the middle of the apparatus would prevent the light to reach the sensor. After the stimulus is given, subject moving hand or foot at east for $1 \mathrm{~cm}$ would close the circuit and the measurement is taken.

Measurements were taken in a dimly lit and silent room. First measurements were taken with the sound stimuli. After right hand/left hand auditory measurements, left hand/right hand light measurements were taken. Then, feet measurements were taken. The experiment was completed after right foot/left foot light measurements and left foot/right foot sound measurements were implemented. The stimuli were initiated by the researcher in random intervals; constant intervals were avoided. Before the actual measurement, participants were informed about the procedure, the sound and the light were introduced and a trial was conducted.

10 different measurements were taken for each stimulus (sound/light) and for each extremity. Maximum 2 values and minimum 2 values among these 10 measurements were ignored and the mean of the remaining 6 measurements was accepted as the reaction time. A total of 80 stimuli were given to each subject within 3 minutes.

Reaction times were compared among the two groups based on left-right, hand-foot and light-sound.

\section{Selective Action Array}

Selective action array was implemented using a table tennis robot (Butterfly/Amic-3000). The robot was set up to send a total of 120 balls in different colors (white, yellow and pink, 40 each) to different points on the table, with the same speed, in random colors and 90 balls per minute. The subjects were asked to ignore the white balls, to touch the yellow balls and to hold the pink balls after the balls hit the table once and using their dominant hands. It was made sure that the subjects did not witness the experiment previously. After the initial test, the second experiment was recorded on video.

The actions that the subjects committed or did not commit were transferred into points using a scoring 
scale within the range of 0 to 6 points (Table 1 ). Results were given as total points and the percentage success of the action worth 6 points for each color of ball, namely " 6 points success percentage."

\section{Statistics}

All statistical analyses were performed with IBM SPSS V. 22.0. The results were given as mean \pm standard deviation, and minimum and maximum values. Shapiro Wilk test was used as normality test. Comparisons of intra-group dependent variables are conducted using Wilcoxon signed rank test when data were not normally distributed. Comparisons of intergroup continuous variables were conducted using Mann-Whitney $U$ test when the data were not normally distributed. Significance level was accepted as $\mathrm{p}<0.05$.

\section{Results}

Mean age for the groups, hand/foot preferences and the period that the subjects actively played computer games or engaged in sports were presented in Table 2.

There was no significant difference between the groups based on their age and the period that they actively played computer games or were engaged in sports.

Reaction times are displayed in Table 3. The average of 8 reaction times given to each extremity and stimulus was given as "mean reaction time".

The most rapid reactions were those given to sound using hands, while the lowest ones were given to light using left foot. Values fewer than average $200 \mathrm{~ms}$ were obtained in 5 of the total 8 different reaction times for both groups. There was no significant difference between the reactions of the subjects given to sound with their dominant hands, and right or left hand sound reaction times.

Mean reaction times for both groups were very similar (TT Group 196.8 ms; CG Group 196.0 ms).

Comparisons conducted within groups are displayed in Tables 4 and 5. Right side was found as more rapid in reactions given to sound using foot in $\mathrm{CG}$ group and given to light using hand in TT group. There was no difference between right and left sides in other comparisons.

In total, reactions given with hand were more rapid than reactions given with foot with an average 15.7 ms. Reactions given to sound were more rapid than reactions given to light with an average $20 \mathrm{~ms}$.

There was no significant difference between table tennis athletes and computer game players based on the reaction times for each extremity or stimulus.

Table 6 demonstrates the total points obtained in selected action array and 6 points success percentages for each colored ball. No significant difference was observed between the groups.

While table tennis athletes demonstrated the best success rates with yellow and pink balls, computer game players were most successful with the white ball.

Table 1: Scoring Scale.

\begin{tabular}{|l|c|}
\hline White Ball > No reaction & Points \\
\hline No reaction & $\mathbf{6}$ \\
\hline Reacted but did not touch & $\mathbf{4}$ \\
\hline Touched & $\mathbf{2}$ \\
\hline Held & $\mathbf{0}$ \\
\hline & \\
\hline Yellow Ball $>$ Touch & $\mathbf{0}$ \\
\hline No reaction & $\mathbf{4}$ \\
\hline Reacted but could not touch & $\mathbf{6}$ \\
\hline Touched & $\mathbf{2}$ \\
\hline Held & \\
\hline & \\
\hline Pink Ball $>$ Hold & $\mathbf{0}$ \\
\hline No reaction & $\mathbf{2}$ \\
\hline Reacted but could not touch & $\mathbf{4}$ \\
\hline Reacted but could not hold & $\mathbf{6}$ \\
\hline Held & \\
\hline
\end{tabular}

Table 2: General characteristics of computer game players (CG) and table tennis athletes (TT).

\begin{tabular}{|lcc|}
\hline Groups & CG Players & TT Athletes \\
\hline $\mathbf{n}$ & 10 & 9 \\
Age (year) & $19.6 \pm 1.5$ & $20.1 \pm 4.9$ \\
& $(18-22)$ & $(15-30)$ \\
Active time (year) & $10.6 \pm 3.4$ & $12.3 \pm 4.8$ \\
Left hand & $(6-15)$ & $(5-20)$ \\
Left foot & 1 & 2 \\
\hline
\end{tabular}


Table 3: Reaction times. (Red areas depict minimum values, blue areas depict maximum values, and green areas depict values below $200 \mathrm{~ms}$.) CG: Computer game players group; TT: Table tennis athletes group.

\begin{tabular}{|c|c|c|c|c|c|}
\hline & & & & CG & TT \\
\hline \multirow{8}{*}{ 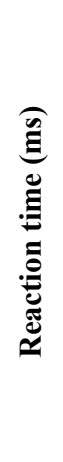 } & \multirow{4}{*}{ 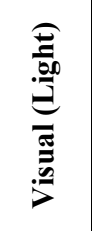 } & \multirow{2}{*}{ Hand } & Right & $201.0 \pm 20.2(172.0-239.7)$ & $189.9 \pm 7.8(179.8-202.5)$ \\
\hline & & & Left & $198.4 \pm 15.5(182.8-221.3)$ & $200.8 \pm 13.2(178.8-218.0)$ \\
\hline & & \multirow{2}{*}{ Foot } & Right & $213.3 \pm 13.7(182.5-228.2)$ & $212.0 \pm 10.7(191.8-232.5)$ \\
\hline & & & Left & $215.2 \pm 7.3(207.8-228.2)$ & $220.5 \pm 14.5(199.7-246.5)$ \\
\hline & \multirow{4}{*}{ 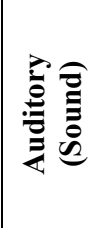 } & \multirow{2}{*}{ Hand } & Right & $176.1 \pm 15.2(156.5-203.7)$ & $180.0 \pm 10.0(166.3-191.3)$ \\
\hline & & & Left & $183.1 \pm 21.1(151.2-210.7)$ & $179.0 \pm 17.4(159.5-208.8)$ \\
\hline & & \multirow{2}{*}{ Foot } & Right & $184.9 \pm 13.9(168.0-215.5)$ & $196.1 \pm 16.9(175.7-219.5)$ \\
\hline & & & Left & $196.1 \pm 19.7(171.7-232.3)$ & $195.8 \pm 16.4(175.0-228.2)$ \\
\hline \multicolumn{4}{|c|}{ Dominant Hand / Sound } & $177.5 \pm 15.5(156.5-203.7)$ & $182.5 \pm 10.3(166.3-196.2)$ \\
\hline \multicolumn{4}{|c|}{ Mean Reaction Time } & $196.0 \pm 12.8(175.6-212.5)$ & $196.8 \pm 9.6(182.6-212.9)$ \\
\hline
\end{tabular}

Table 4: Intra-group comparisons among the computer game players (CG) group.

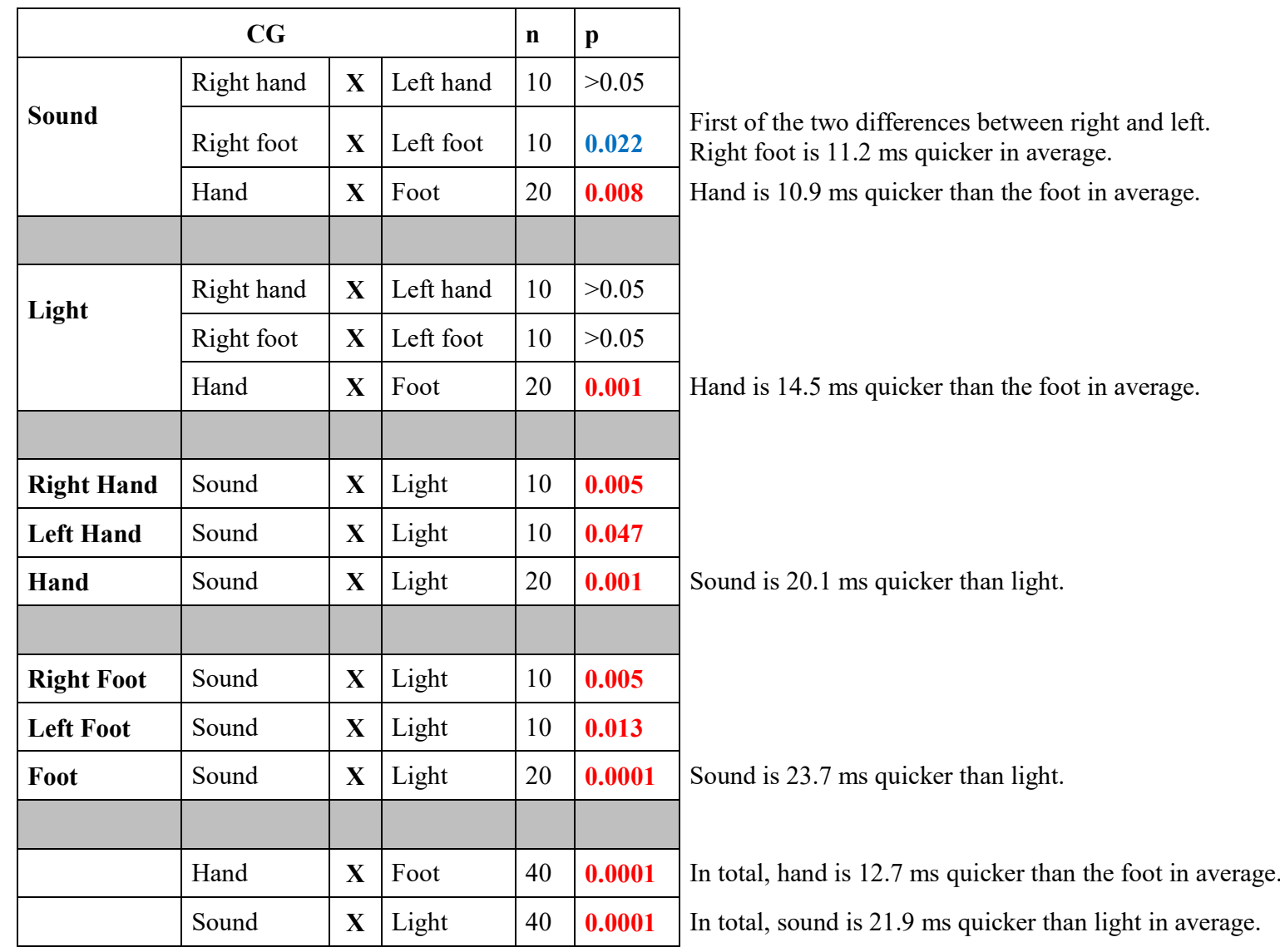


Table 5: Intra-group comparisons in table tennis athletes (TT) group

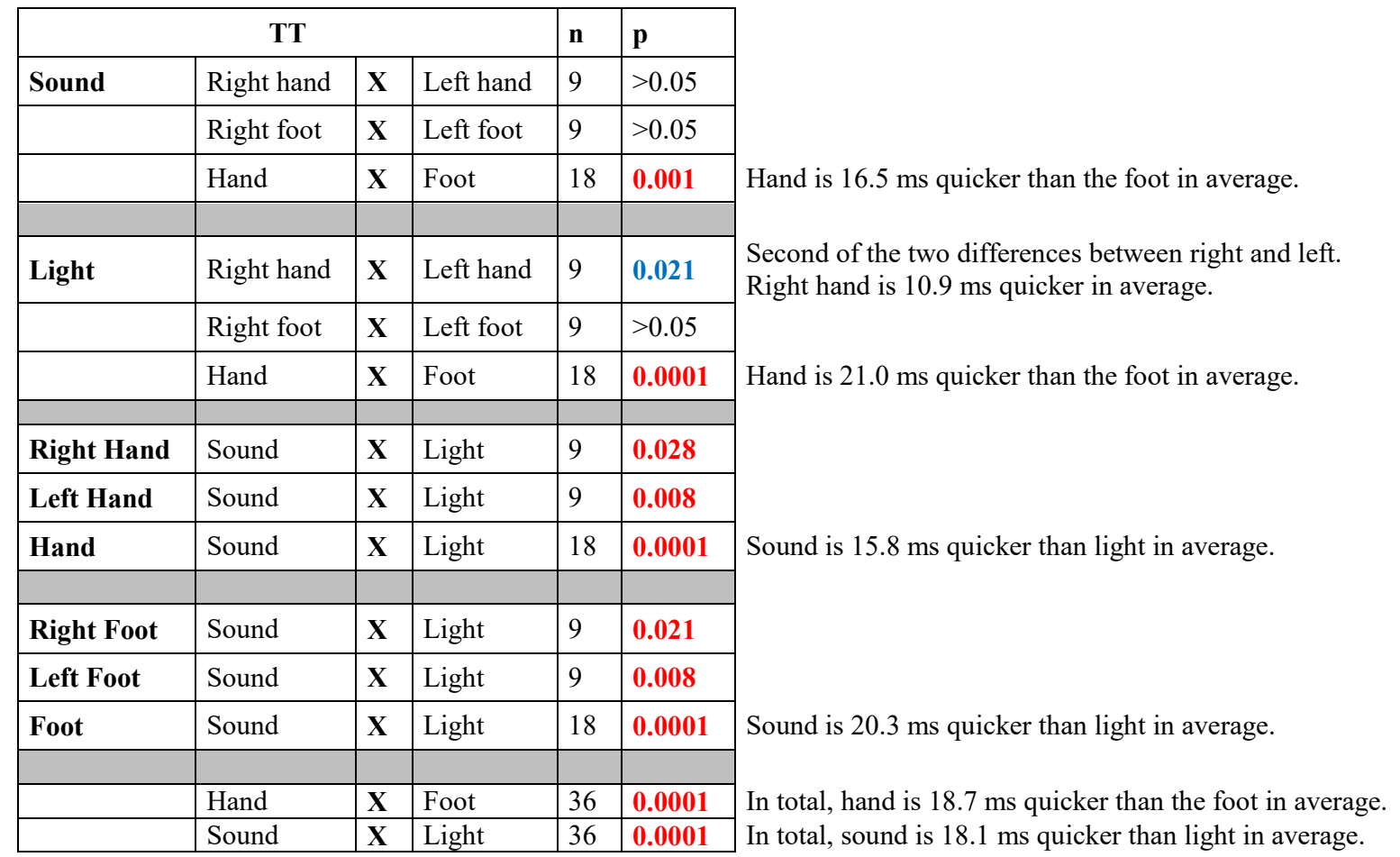

Table 6: Total points and 6 points success percentages obtained in selective action array. CG: Computer game players group; TT: Table tennis athletes group.

\begin{tabular}{|c|c|c|c|c|}
\hline \multirow{2}{*}{} & \multirow{2}{*}{ Total point } & \multicolumn{3}{|c|}{ 6 points success percentage } \\
\cline { 3 - 5 } & & White & Yellow & Pink \\
\hline CG & $156.3 \pm 10.1(139.6-167.8)$ & $90.0 \pm 7.9(73.1-100.0)$ & $79.8+10.7(58.1-92.6)$ & $56.6 \pm 21.4(8.3-79.4)$ \\
\hline TT & $158.1 \pm 10.8(140.6-172.8)$ & $87.6 \pm 10.3(69.7-100.0)$ & $81.3 \pm 11.5(53.3-93.3)$ & $68.8 \pm 17.7(44.4-89.3)$ \\
\hline
\end{tabular}

\section{Discussion}

In this study, where the accuracy rates for the selective reactions and reaction times of computer game players and table tennis athletes were compared, the reaction times of athletes and players were found to be quite similar. For the selective action array where the selective reactions were evaluated, table tennis athletes obtained the best scores in total points and points with the yellow and pink balls, computer game players had more success with the white balls.

Measurement of the reaction times under 8 different conditions puts this study in a unique situation among other studies (3-6). Evaluation of all extremities both visually and aurally and obtaining a mean reaction time made it possible to analyse the subjects as a whole. The subjects went below the mean value of $200 \mathrm{~ms}$ in 5 of 8 different situations in both groups.

Medical Science and Discovery 2016; 3(2): 91-7
Right hand-light reaction time for table tennis athletes was significantly shorter than left hand-light reaction time. 7 out of 9 table tennis athletes had a dominant right hand. In table tennis sport, the ball approaching from the opposite side is perceived visually and the reaction is given using the racket. Naturally, a more rapid response while using the dominant side could be expected.

However, the lack of such a difference in reactions given as a response to sound stimuli demonstrates that visual perception is dominant in the sport of table tennis.

The second significant difference between the right and left sides was observed in the reactions given by the right foot as a response to sound stimuli among the computer game players when compared to the left side foot. It was difficult to explain this difference of 11 ms. 
This difference could be explained by the existence of computer games that assign a special function especially to the right foot. However, although this situation was questioned in the study, this occurrence was not identified.

The fact that the standard deviations for the right hand reaction times for both auditory and visual stimuli by table tennis athletes had the lowest values when all analyses were compared demonstrated without doubt that they could concentrate consistently for longer periods of time and the effects of the sports on the hand utilized for these athletes, most of which were right-handed. The significance of visual perception in both the sport of table tennis and computer gaming is obvious. The right-hand visual reaction times demonstrated that table tennis athletes were an average of $11 \mathrm{~ms}$ more quick than the computer game players $(189.9 \pm 7.8 ; 201.0 \pm 20.2)$. This could be explained by the effects of the sport on muscle coordination.

Several studies demonstrated that table tennis athletes had lower reaction times when compared to other racket sports' athletes and individuals that do not participate in sports (3-6). However, the findings of this study did not reflect a significant difference between the reaction times of table tennis athletes and computer game players. This result shows the skills of computer game players in maintaining the game they play by concentrating on the immediate action, just like an athlete, using responses to minute visual and auditory stimuli on the computer screen and by implementing the accurate action with the accurate timing. It was considered that by keeping the attention and readiness of the player intact continuously, this process enables the players to maintain their reaction times and responses to the selective reaction dynamically.

While the similarity of the reaction times of the computer game players and table tennis athletes was an expected finding, the similarity in achievement scores in selective action array was not. The finding that the computer game players achieved better scores with the white balls and table tennis athletes achieved better scores with yellow and pink balls demonstrated that the array utilized in this study was set up accurately. But the lack of difference could be due to the limited number of subjects. The fact that table tennis athletes react to each and every ball that their opponent sends was totally contradictory to the action they should take with the white balls in the array set up in the study. Computer game players recognized this difference better than the athletes did. However the action requested by the subjects for the yellow and especially the pink balls was a more difficult one to perform accurately. Table tennis athletes, who are aware of the exact bounce rates of the ball and with better coordination, performed better with the yellow and especially the pink ball, albeit not significantly.

The gradual nature of the success rates for different coloured balls, and the highest success rates with the white balls and the lowest success rates with the pink balls demonstrated the difference between the degrees of difficulty between the balls with different colours. Based on the degree of difficulty of the action, white ball was the easiest, yellow ball was with medium level of difficulty and the pink ball was the hardest.

The reaction time and selective action array applications used in the study required very high levels of attention and concentration within a very short period of time. After an interruption during the application, it was very difficult to concentrate again. Reaction time is closely related to attention and concentration. Thus, the most significant effect of training in competitive sports is to learn how to concentrate and focus in the game. Reaction time in sports is considered as a motor trait that could be developed via training (12-14). Effect on the cognitive function of computer games is a fact now accepted $(8,15,16)$.

\section{Conclusion}

The findings of this study demonstrated expected reaction times and unexpectedly good selective action array achievements levels with computer game players. This result could be interpreted as computer games and/or advanced computer programs developed for sports could support to decrease reaction times, to increase visual perception and attention and to retain concentration for extended periods of time in training of the athletes. By affiliating appropriate computer games/software with training programs, in addition to improving cognitive features such as reaction time, attention, concentration and visual perception, rapid and accurate adaptation to new situations and the skill to develop strategies could be achieved among athletes. Especially in injury and rehabilitation periods of the athletes, computer games/software could be used to retain existing capabilities.

Acknowledgements: This study was presented as a oral presentation in the "9th International Computer Sciences in Sports Congress" (June 19-22, 2013 Istanbul, TURKEY)

This study was supported by Uludağ University Scientific Research Projects Department [KUAP (E) 2013/57].

Conflict of Interest: The authors declare no potential conflicts of interest with respect to the research, authorship, and/or publication of this article. 


\section{References}

1. Lees A. Science and the major racket sports: a review. J Sports Sci.2003;21(9):707-32.

2. Tang H, Mizoguchi M, Toyoshima S. Speed and spin characteristics of the $40 \mathrm{~mm}$ table tennis ball. International Journal of Table Tennis Sciences. 2002;4-5:278-84.

3. Akpinar S, Devrilmez E, Kirazci S. Coincidenceanticipation timing requirements are different in racket sports. Percept Mot Skills. 2012;115(2):581-93.

4. Ak E, Koçak S. Coincidence-anticipation timing and reaction time in youth tennis and table tennis players. Percept Mot Skills. 2010;110(3):879-87.

5. Mouelhi Guizani S, Tenenbaum G, Bouzaouach I, Ben Kheder A, Feki Y, Bouaziz M. Information-processing under incremental levels of physical loads: comparing racquet to combat sports. J Sports Med Phys Fitness. 2006;46(2):335-43.

6. Bhabhor MK, Vidja K, Bhanderi P, Dodhia S, Kathrotia R Joshi V. A comparative study of visual reaction time in table tennis players and healthy controls. Indian J Physiol Pharmacol. 2013;57(4):439-42.

7. Hutchins B. Signs of meta-change in second modernity: the growth of e-sport and the World Cyber Games. New Media Society. 2008;10(6): 851-69.

8. Granic I, Lobel A, Engels RC. The benefits of playing video games. Am Psychol. 2014;69(1):66-78.
9.

Funk JB. Video 2005;16(2):395-411.

10. Crawford G. Digital Gaming, Sport and Gender. Leisure Stud. 2005;24(3):259-70.

11. Jonasson K, Thiborg J. Electronic sport and its impact on future sport. Sport in Society: Cultures, Commerce, Media, Politics. 2010:13(2):287-99.

12. Iida Y, Miyazaki M, Uchida S. Developmental changes in cognitive reaction time of children aged 6-12 years. Eur J Sport Sci. 2010;10(3):151-8.

13. Mori S, Ohtani Y, Imanaka K. Reaction times and anticipatory skills of karate athletes. Hum Movement Sci. 2002;21:213-30.

14. Schwab S, Memmert D. The impact of a sports vision training program in youth field hockey players. J Sport Sci Med. 2012;11:624-31.

15. Latham AJ, Patston LL, Tippett LJ. The virtual brain: 30 years of video-game play and cognitive abilities. Front Psychol. 2013;4:629.

16. Oei AC, Patterson MD. Enhancing cognition with video games: a multiple game training study. PLoS One. 2013;8(3):e58546.

Copyright (C) 2014 The Author(s); This is an open-access article distributed under the terms of the Creative Commons Attribution License (http://creativecommons.org/licenses/by/4.0), which permits unrestricted use, distribution, and reproduction in any medium, provided the original work is properly cited. All Rights reserved by international journal of Medical Science and Discovery. 